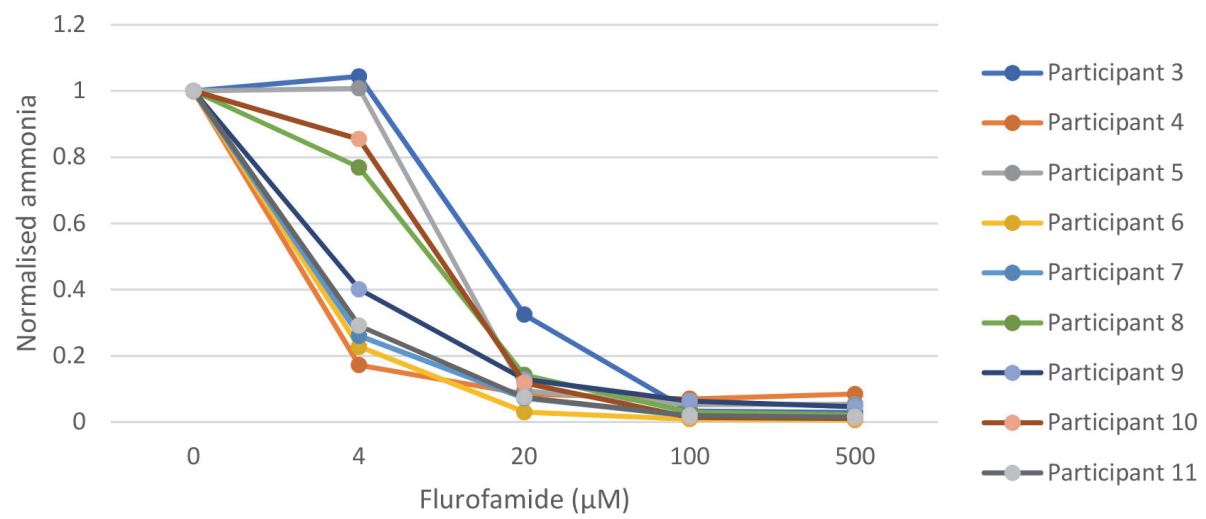

Abstract P096 Figure 1 Ammonia production, normalised to level with no flurofamide present

(300 $\mathrm{mM}$ ) in the medium used, but urea fell in cultures without flurofamide relative to cultures with higher concentrations of the compound.

Conclusion In an in vitro system, flurofamide inhibited virtually completely the production of ammonia from urea by colonic microorganisms; this occurred at concentrations which might feasibly be obtained in vivo.

\section{P097 ACUTE VARICEAL BLEEDING IN A LARGE TEACHING HOSPITAL: A 5-YEAR ANALYSIS}

Anju Phoolchund*, Megha Bhandari, Luigi Medri, Srishti Sarkar, Chanice Bloomfield, Philip Oppong, Puneet Chhabra, Philip Boger, Praful Patel, Imdadur Rahman. Southampton Interventional Endoscopy Unit, University Hospital Southampton, Southampton, UK

\subsection{6/gutjnl-2021-BASL. 105}

Acute variceal bleeding (VB) remains a major cause of death in cirrhosis/portal hypertension. We aim to assess the characteristics, therapies delivered and outcomes of patients presenting with $\mathrm{VB}$ in a large teaching hospital.

A retrospective analysis was performed on all patients presenting with acute UGIB who underwent a gastroscopy between March 2015 and February 2020. Data was collected where UGIB was deemed secondary to varices from electronic records to assess findings at endoscopy, therapy applied, rebleed rates and mortality.

A total of 2324 patients presented with UGIB. 239 presentations involving 196 patients had varices reported. 155 cases underwent therapy for acute VB as cause for UGIB, with varices felt not to be the primary cause in the remaining 84 . Median age was 54 years(IQR 46-67). 65\%(n=100) were male. Clinical presentations were haematemesis only $35 \%$ $(n=55)$, melaena only $26 \%(n=41)$ or both $38 \%(n=59)$. $56 \%$ $(n=87)$ gastroscopies were undertaken 'out of hours'. The commonest endoscopic finding was oesophageal varices in $98 \%(n=152)$.

Treatment was undertaken at first endoscopy in $93 \%$ $(\mathrm{n}=144)$, comprising banding $(86 \%)$ and glue injection(7\%). $17 \%(n=27)$ of patients required insertion of Sengstaken-Blakemore tube. The remaining $7 \%(n=11)$ underwent their first treatment at further gastroscopy (within 7 days).

$32 \%(n=49)$ of patients had repeat gastroscopy within 7 days, where $90 \%$ were due to clinical evidence of rebleed. 23 patients required repeat endoscopic therapy and 10 patients underwent Sengstaken-Blakemore tube insertion.
$30 \%(n=70)$ of patients required ICU admission. $16 \%$ $(\mathrm{n}=25)$ underwent TIPS procedure.

30 -day rebleeding rate was $24 \%(n=37) .30$-day mortality was $17 \%(n=27)$ with $67 \%$ attributable to UGIB or subsequent decompensated cirrhosis with multi-organ failure.

On subgroup analysis, ICU admission was significantly associated with increased mortality (OR 3.46, 95\% CI 1.46 to 8.22, $\mathrm{p}<0.01$ ) but rebleeding (OR 1.35, 95\% CI 0.54 to $3.23, \mathrm{p}=0.53$ ) or having a gastroscopy 'out of hours' (OR $1.48,95 \%$ CI 0.55 to $3.95, \mathrm{p}=0.43$ ) were not.

Receiver operating characteristic (ROC) curves showed better predictive ability of MELD score than Child-Pugh score for 30-day mortality (AUC 0.92 vs 0.72 ), with MELD score of $>18$ significantly associated with increased mortality (OR $7.70,95 \%$ CI 0.04 to $0.35, \mathrm{p}<0.01$ ) similar to published data.

Mortality and rebleed rates following acute VB is in keeping with national data. More than half of patients undergo gastroscopy out of hours emphasizing the need for 24/7 access to therapeutic endoscopy. MELD score of $>18$ appears to be a strong predictor of mortality and can be used in clinical practice to consider early critical care involvement.

\section{P098 CIRRHOCARE ${ }^{\circledR}$-A PILOT STUDY OF DIGITAL HOME MANAGEMENT OF ADVANCED CIRRHOSIS TO DETERMINE FEASIBILITY AND UTILITY TO DIAGNOSE NEW DECOMPENSATION EVENTS}

${ }^{1,2}$ Konstantin Kazankov*, ${ }^{1}$ Devnandan Amor Chatterjee, ${ }^{1}$ Simone Novelli, ${ }^{1}$ Alexandra Phillips, ${ }^{3}$ Anu Balaji, ${ }^{3}$ Maruthi Raja, ${ }^{1,3}$ Rajiv Jalan, ${ }^{3}$ Ravan Boddu, ${ }^{3}$ Ravi Kumar, 1,2Rajeshwar P Mookerjee. 'Liver Failure Group, Institute for Liver and Digestive Health, UCl Medical School, Royal Free Hospital, London, UK; ${ }^{2}$ Department of Hepatology and Gastroenterology, Aarhus University Hospital, Aarhus, Denmark; ${ }^{3}$ Cyberliver Limited, Manchester, UK

\subsection{6/gutjnl-2021-BASL.106}

Background and Aims Patients discharged from hospital following acute decompensation are at high risk of new complications and need close follow-up, limited currently by the growing burden of cirrhosis and impact of COVID-19. Specialist liver care in the community is an unmet need, to reduce hospital exposure and manage new decompensation events.

Methods We included 20 patients with cirrhosis and recent acute decompensation. Commercially available devices and a smartphone (+SIM card) were given to all patients for 
daily recording of ECG, blood pressure, weight, and\% body-water (bioimpedance), Stroop test (hepatic encephalopathy (HE) assessment), and self-reported well-being and food/fluid/alcohol intake. Data was Blue-toothed via a secure server to the CirrhoCare ${ }^{\circledR}$-App, which had 2-way patient-physician communication. Hepatologists evaluated daily data and facilitated interventions as required. A matched control cohort $(n=20)$ with advanced cirrhosis was observed in parallel.

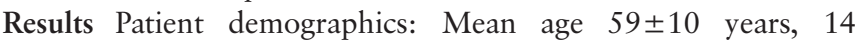
male, main etiology alcohol (75\%); 75\% Child-Pugh class B. Fifteen patients $(75 \%)$ showed good compliance, $(\geq 4$ readings/ week), 2 had moderate compliance (2-4/week), and 3 had poor compliance $(<2 /$ week). In a usability questionnaire scored $1-10$, the median score was $\geq 9$ for any given question.

Mean follow-up was $10.1 \pm 2.4$ weeks. Amongst CirrhoCare ${ }^{\circledR}$ managed patients, 1 died and 1 received a liver transplant. Eight readmissions occurred in 5 different patients: 3 due to $\mathrm{HE}, 1$ to acute-kidney injury (AKI), 1 to both AKI and HE, and 3 in the same patient to rectal bleeding. The median readmission lasted 5 (IQR 3.5-11) days, and none was $>14$ days. Except for the acute bleeds, we identified early signs of decompensation in all cases, e.g. failed Stroop test, hypotension or reduction/gain in body fluid (weight), and facilitated 2 short hospitalizations of the 8 total readmissions.

Based on early signs of decompensation, we contacted patients on 16 other occasions, guiding intervention and likely preventing further readmissions as confirmed by an independent physician panel. Two controls died during follow-up, and there were 13 readmissions in 8 patients, lasting median 7 (IQR 3-15) days with four admissions $>14$ days. They had 6 unplanned paracenteses compared to 1 in CirrhoCare ${ }^{\circledR}$-managed patients.

Conclusions CirrhoCare ${ }^{\circledR}$ 's novel, multimodal, home-monitoring in patients with advanced cirrhosis is feasible with excellent patient engagement, and prompts early diagnosis of decompensating events and their intervention; and hospital admissions are fewer and shorter in duration than in controls. We propose the application of CirrhoCare ${ }^{\circledR}$ for assisted, specialist, community management of advanced cirrhosis.

\section{P099 SERUM GAMMA GLUTAMYL TRANSFERASE ( $\gamma$ GT) - A PROGNOSTIC BIOMARKER FOR DETRIMENTAL NEUTROPHILS IN PATIENTS WITH HEPATOCELLULAR CARCINOMA (HCC)?}

${ }^{1}$ Marcello Kadharusman, 'Misti McCain, 'Joao Mauricio, 'Maja Laszczewska, 'Jack Leslie, ${ }^{1}$ Daniel Geh, ${ }^{1}$ Derek Mann, ${ }^{1}$ Ruchi Shukla, ${ }^{1,2}$ Helen L Reeves*. ' Newcastle University, Newcastle upon Tyne, UK; ${ }^{2}$ Newcastle upon Tyne Hospitals NHS Foundation Trust, Newcastle upon Tyne, UK

\subsection{6/gutjnl-2021-BASL.107}

Background Deaths from HCC are rising dramatically in the UK. Immuno-oncology therapies herald a new era of treatment, but only a minority of patients respond. Elevated circulating neutrophils are associated with a poor prognosis, although mechanisms are poorly understood and clinically useful biomarkers of 'poor prognostic neutrophils' are lacking. Serum $\gamma$-GT recognised as a biomarker of a neutrophil inflamed liver microenvironment. As a membrane-bound enzyme it promotes intracellular glutathione, limiting cellular damage. It may also promote cancer cell survival.

Methods Serum $\gamma$-GT associations were explored in 300 patients with HCC. Correlations with tissues expression and CD66b +ve neutrophils were explored in a subset of 50 patients.

Results There were highly significant positive correlations $(<0.01)$ between serum $\gamma$-GT and deteriorating ECOG PST and presence of portal vein thrombosis (PVT). Serum $\gamma$-GT correlated negatively with albumin (Pearson -0.134, $\mathrm{p}=0.020$ ) and positively with bilirubin $(0.124, \mathrm{p}=0.032)$, neutrophils (0.221, $\mathrm{p}<0.0001)$ and platelets $(0.171, \mathrm{p}=0.003)$. In a multivariate cox regression analysis, ECOG PST, albumin, PVT, platelets and $\gamma$-GT were independently associated with poorer survival. $\gamma$-GT expression is typically on the canalicular surface of hepatocytes. In a subset of patients with tissues available, immunohistochemistry confirmed an elevation in expression intensity in up to $50 \%$ of patients with HCC compared to cirrhotic controls without cancer. In addition, aberrant membranous and cytoplasmic $\gamma$-GT expression was acquired in $30 \%$ of cancer cases - in tumour as well as peri-tumour liver. It was the cytoplasmic $\gamma$-GT in peri-tumour liver that correlated significantly with declining PST, Childs Pugh score and PVT. Tissue neutrophils were detected with CD66b immunohistocehmistry. These were elevated in peri-tumour areas, associated with poorer response to treatment and outcome. Elevation in $\mathrm{CD} 66 \mathrm{~b}+$ neutrophils was associated with the aberrant cytoplasmic $\gamma$-GT (See figure 1). Studies are ongoing, but $\mathrm{CD} 66 \mathrm{~b}$ positive neutrophils in the peri-tumour area correlated with serum $\gamma$-GT in a highly significant fashion $(0.838$, $\mathrm{p}=0.001$, Spearmans Rho, $\mathrm{n}=11$ ).

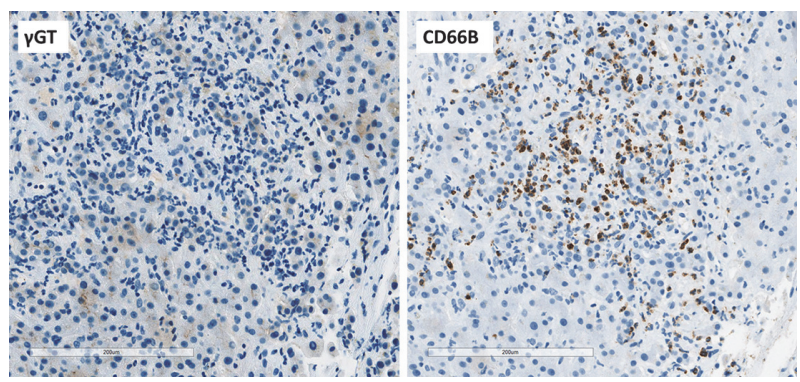

Abstract P099 Figure 1 Aberant cytoplasmic $\gamma \mathrm{GT}$ in peri-tumour liver, with CD66b+ neutrophils

Conclusion/Discussion Serum $\gamma$-GT correlates significantly with invasive/advanced HCC, deteriorating ECOG PST and circulating neutrophils and is independently associated with poorer survival. In tissues, aberrant cytoplasmic $\gamma$-GT is present in the peri-tumour liver, enriched with CD66b positive neutrophils, both associated with progressive tumour features and serum $\gamma$-GT. Ongoing studies will define the neutrophil phenotype associated with aberrant $\gamma$-GT expression, while exploring serum $\gamma$-GT as a predictive biomarker, guiding immuno-oncology combination therapies. Combinations targeting pro-tumour neutrophils in combination with those targeting $\mathrm{T}$ cells may increase responses to immunooncology therapies. 